\title{
Pregnancy in Women with Atypical Hemolytic Uremic Syndrome
}

\author{
Eric Rondeau $^{a} \quad G^{2}$ ianluigi Ardissino ${ }^{b}$ Marie-Pierre Caby-Tosi ${ }^{c}$ \\ Imad Al-Dakkak ${ }^{d}$ Fadi Fakhouri $^{\text {e }}$ Benjamin Miller $^{f}$ Marie Scully ${ }^{g}$ \\ on behalf of the Global aHUS Registry
}

antensive Care Nephrology and Transplantation Department, Hôpital Tenon, APHP, Sorbonne Université, Paris, France; ${ }^{b}$ Centro per la Cura e lo Studio della Sindrome Emolitico-Uremica, Fondazione IRCCS Ca' Granda Ospedale Maggiore Policlinico, Milan, Italy; ' ${ }^{C}$ lobal Data Science, Alexion Pharmaceuticals, Inc., Boston, MA, USA;

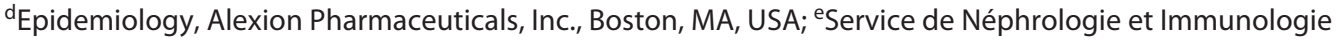
Clinique, CHU de Nantes - Hôtel Dieu, Nantes, France; ${ }^{f}$ Employee at the Time of Study, of Alexion Pharmaceuticals, Inc., Boston, MA, USA; ${ }^{9}$ Department of Haematology, University College London Hospital, Cardiometabolic

Programme-NIHR UCLH/UCL BRC, London, UK

\section{Keywords}

Atypical hemolytic uremic syndrome · Eculizumab ·

Pregnancy $\cdot$ Real-world evidence $\cdot$ Registry

\section{Abstract}

Background: Pregnancy outcomes in patients with atypical hemolytic uremic syndrome (aHUS) are not well-documented. Here, we present characteristics of and outcomes for patients with aHUS who became pregnant while enrolled in the Global aHUS Registry. Methods: The observational Global aHUS Registry (NCT01522183), initiated in April 2012, collects demographics, disease history, treatment, and outcomes data for patients with aHUS, regardless of treatment approach. This descriptive analysis includes patients from the Registry with evaluable pregnancy data supplemented with pharmacovigilance information; the number of pregnancies, outcomes, and exposure to eculizumab were evaluated. $\boldsymbol{R e}$ sults: As of April 1, 2019, 44 pregnancies were recorded in 41 patients, with 24 pregnancies exposed to eculizumab. Pathogenic variants were identified in $48.8 \%$ of patients. Three patients were on dialysis and 6 patients had a kidney graft at the time of pregnancy. Excluding elective terminations, $85.3 \%$ of pregnancies resulted in live births. Elective terminations were recorded in $22.7 \%$ of pregnancies, miscarriages occurred in $9.1 \%$ of pregnancies, and late fetal death in $2.3 \%$ of pregnancies. No malformations or anomalies were reported. Conclusions: Our results show that in women with aHUS, even on dialysis or with a kidney graft, pregnancy is possible with careful monitoring for aHUS flares and prematurity. Prophylactic or therapeutic eculizumab offers disease control with low-risk of fetal abnormalities.

(c) 2021 The Author(s).
Published by S. Karger AG, Basel

\section{Introduction}

Atypical hemolytic uremic syndrome (aHUS) is a disease caused by dysregulation of alternative complement pathways leading to systemic thrombotic microangiopathy (TMA) and associated damage to the kidneys and

The members of the Global aHUS Registry are listed in the Acknowledgements.
C 2021 The Author(s).

Published by S. Karger AG, Basel

This is an Open Access article licensed under the Creative Commons Attribution-NonCommercial-4.0 International License (CC BY-NC) (http://www.karger.com/Services/OpenAccessLicense), applicable to the online version of the article only. Usage and distribution for commercial purposes requires written permission.
Correspondence to:

Eric Rondeau, eric.rondeau@tnn.aphp.fr 
other organs [1,2]. Renal impairment is a severe complication of aHUS; for instance, $47 \%$ of adults and $17 \%$ of children progressed to end-stage renal disease or death during the first manifestation of aHUS and, after 1 year, the proportion further increased to $56 \%$ of adults and $29 \%$ of children [3]. Pregnancy in patients with aHUS is considered high risk because some patients have underlying chronic kidney disease (CKD) and aHUS recurrence during or after pregnancy is a possibility. Adverse pregnancy outcomes in high-risk patients with CKD are significantly increased due to risk factors including kidney dysfunction, proteinuria, hypertension, and poorly controlled disease [4,5]. Qualitative evidence shows that pregnancy in patients with CKD may be discouraged by the physician or patients themselves decide against becoming pregnant due to the possibility of adverse outcomes [6].

Data on maternal and fetal outcomes in women with a prior diagnosis of aHUS are emerging [7-11]. A recently published pharmacovigilance report describes outcomes of live births, pregnancy failure, induced abortion, and maternal death in pregnant women with aHUS and treated with eculizumab $(n=99)$ [7]. In this cohort, $59 \%$ of pregnancies resulted in live births, $20.4 \%$ in elective terminations, $14.8 \%$ in spontaneous abortion, and $5.6 \%$ in fetal death, with no maternal deaths reported. Although it involved a large cohort of patients, the report was based on a pharmacovigilance database; relevant patient medical history, including dialysis or transplantation status, kidney function (estimated glomerular filtration rate), family history of aHUS, and genetics, was not characterized systematically. Also, gestational age and birth weight were not reported.

In this analysis based on Global aHUS Registry data, we describe characteristics in women with aHUS who became pregnant after the diagnosis, including those considered high-risk for pregnancy. Additionally, we also analyze pregnancy outcomes in this population.

\section{Methods}

\section{Study Design}

The Global aHUS Registry (NCT01522183), initiated in 2012, is an observational, noninterventional, multicenter, multinational study of patients with aHUS from participating sites. It is a large, real-world database on demographics, medical history, treatment, and outcomes data for patients with aHUS [12]. The Registry collects both retrospective and prospective data in patients with a clinical diagnosis of aHUS, with follow-up every 6 months. Prior to study onset, approval was obtained from the Ethics Committees and institutional review boards of all participating centers. Inves- tigators conducted all aspects of this study in accordance with all national, provincial, and local laws of the pertinent regulatory authorities. Written informed consent was provided by patients or their parents or guardians, as deemed applicable by Institutional Review Boards or Independent Ethics Committees.

\section{Patients}

Patients of all ages with a clinical diagnosis of aHUS were eligible for enrollment in the Global aHUS Registry and are not required to have an identified complement gene abnormality or complement factor $(\mathrm{CF}) \mathrm{H}$ autoantibody nor were they required to have previous or ongoing treatment with eculizumab (full description of criteria used for patient enrollment is provided in the online suppl. material). Exclusion criteria were evidence of Shiga toxinproducing Escherichia coli infection, ADAMTS13 activity $\leq 5 \%$, or a subsequent diagnosis of thrombotic thrombocytopenic purpura [12]. At the time of this analysis, the Global aHUS Registry, contained entries for a total of 1,742 patients, 1,111 of whom were adults, and 688 of whom were female. Patients included in this analysis were from the following countries: Australia, Austria, Belgium, Denmark, France, Germany, Italy, Russia, Spain, the UK, and the USA. Eculizumab is approved for the treatment of aHUS in each country from which patients were included.

\section{Data Collection and Analysis}

Women who became pregnant after receiving diagnosis of aHUS and enrollment in the Global aHUS Registry and had evaluable pregnancy data were included in the current analysis (Fig. 1); data cutoff was April 1, 2019. Pregnancy data were extracted from the Alexion pharmacovigilance database (Argus Safety ${ }^{\circledR}$ ) and supplemented with clinical characteristics data from the Registry database (MediData Rave). Per protocol, pregnancy outcome data for all patients were collected during this study, including those with eculizumab exposure during pregnancy. Individuals with a missing pregnancy outcome, an ongoing pregnancy, or no data on eculizumab exposure were excluded. Eculizumab exposure was defined as a time overlap between pregnancy and any eculizumab administration, except for women who had an elective termination with eculizumab exposure $\leq 3$ days prior to termination (who were considered unexposed during pregnancy). Women who had an elective termination of pregnancy with eculizumab exposure $\leq 3$ days prior to termination were analyzed as having no exposure to eculizumab during pregnancy. Pregnancies were stratified by eculizumab exposure status and, as such, the categories were not mutually exclusive at the patient level (i.e., a patient with multiple pregnancies could be categorized as having been both exposed to eculizumab during pregnancy and not exposed to eculizumab during pregnancy, if eculizumab treatment status differed during each pregnancy). Obstetric data analyzed included number of live births, miscarriages, elective terminations, gestational age, and late fetal death. Premature delivery was defined as delivery before 37 weeks of gestation. Birth weight was recorded in cases resulting in a live birth. TMA during pregnancy was defined as reported by the treating physician. Genetic testing was conducted at laboratories local to sites participating in the Registry and choice of genetic tests were at the discretion of the treating physician. All data were analyzed descriptively. Renal function (estimated by the CKD Epidemiology Collaboration formula), proteinuria, and presence or absence of high blood pressure were recorded. 
Fig. 1. Disposition of eligible pregnant

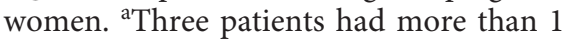
pregnancy.

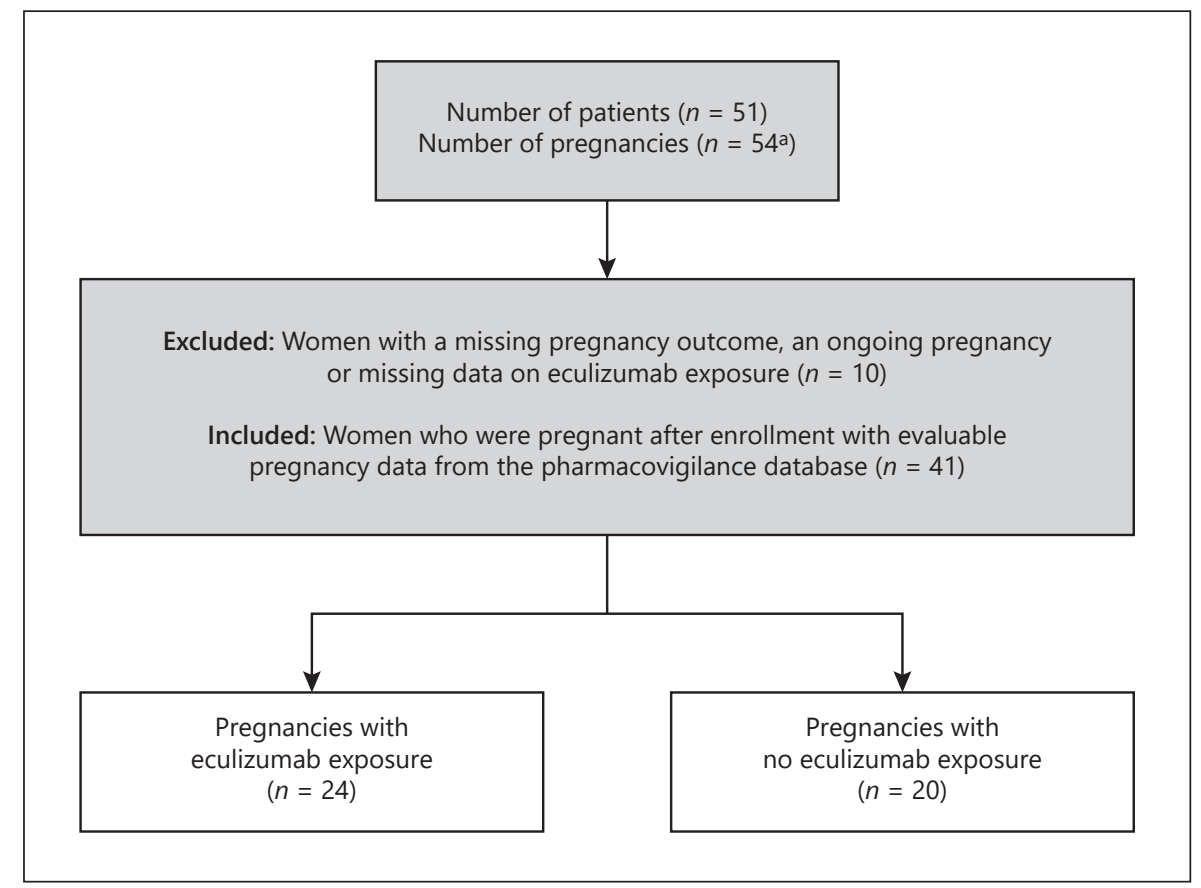

\section{Results}

\section{Patients}

As of April 1, 2019, 54 pregnancies in 51 patients with a previous diagnosis of aHUS were reported in the Global aHUS Registry. Ten patients did not meet the analysis criteria and were excluded (Fig. 1). Forty-four pregnancies in 41 patients were analyzed, with 24 pregnancies exposed to eculizumab (Table 1). The majority of women exposed to eculizumab received standard dosing (1,200 mg every 2 weeks; 19/24 [79.2\%]); of the remainder, dosing was decreased ( $n=3$ [900 mg every 2 weeks, $900 \mathrm{mg}$ every 5 weeks, or $1,200 \mathrm{mg}$ every 18 days $])$, increased ( $n=1$ [1,200-1,500 mg every week]), per physician decision, or data were missing $(n=1)$. No concomitant conditions were identified to suggest TMA of a different etiology to aHUS. Of note, 6 patients were diagnosed with aHUS during, or in the postpartum period of, a prior pregnancy. A combination of hypertension and proteinuria was noted in 5 pregnancies, with 2 specifically noted as pre-eclampsia (1 eculizumab-exposed and 1 nonexposed). Both cases of pre-eclampsia were reported at approximately 31 weeks of gestation; temporal data were unavailable for the 3 remaining cases of hypertension and proteinuria. First pregnancies were recorded in 26 patients. Three patients were on concomitant dialysis during their pregnancies, and 6 patients had a previous kidney transplant.

Pregnancy Outcomes in Women with Atypical Hemolytic Uremic Syndrome

\section{Genetic Analysis}

Thirty-three patients (80.5\%) were analyzed for pathogenic variants of complement regulatory genes (Table 2). Pathogenic variants were identified in 20 of 33 (60.6\%) tested patients; 20 of 41 (48.8\%) patients overall; and 11 of $24(45.8 \%)$ and 9 of $20(45.0 \%)$ pregnancies with and without eculizumab exposure, respectively. No pathogenic variants were found in 10 of 41 (24.4\%) patients tested for at least 5 genes. The most frequent pathogenic variants identified were CFH, CFB, and C3. CFH (12/28 [42.9\%]) variants were detected in a similar proportion of eculizumab-exposed and nonexposed patients. CFB (6/28 [21.4\%]) and C3 (5/25 [20.0\%]) variants were identified mainly in pregnancies with exposure to eculizumab (Table 3; online suppl. Table 1; for all online suppl. material, see www.karger.com/doi/10.1159/000518171). Anti-CFH autoantibodies were detected in 2 of 24 (10.5\%) pregnancies. In both cases, anti-CFH autoantibodies were detected shortly after eculizumab was initiated and both patients had also previously received eculizumab.

\section{Pregnancy Outcomes}

Overall, 29/44 (65.9\%) pregnancies resulted in live birth; excluding elective terminations, live births occurred in 29 of $34(85.3 \%)$ cases, with a similar proportion between eculizumab-exposed and nonexposed pregnancies (Table 4). Out of 23 pregnancies without renal trans- 
Table 1. Baseline characteristics of pregnant patients with aHUS and by exposure to eculizumab

\begin{tabular}{|c|c|c|c|}
\hline Characteristic & $\begin{array}{l}\text { All patients } \\
(N=41)\end{array}$ & $\begin{array}{l}\text { Pregnancy with } \\
\text { exposure to } \\
\text { eculizumab }(n=24)\end{array}$ & $\begin{array}{l}\text { Pregnancy with } \\
\text { no exposure to } \\
\text { eculizumab }(n=20)\end{array}$ \\
\hline Age of mother at diagnosis of aHUS in years, median (range) & $\begin{array}{l}N^{\mathrm{d}}=40 \\
27.6(0.1-38.4)\end{array}$ & $\begin{array}{l}N^{\mathrm{d}}=23 \\
26.9(0.9-38.4)\end{array}$ & $\begin{array}{l}N^{\mathrm{d}}=20 \\
28.2(0.1-38.2)\end{array}$ \\
\hline Childhood onset of aHUS & $4(9.8)$ & $3(12.5)$ & $1(5.0)$ \\
\hline Family history of aHUS ${ }^{a}$ & $\begin{array}{l}N^{\mathrm{d}}=31 \\
5(16.1)\end{array}$ & $\begin{array}{l}N^{\mathrm{d}}=19 \\
3(15.8)\end{array}$ & $\begin{array}{l}N^{\mathrm{d}}=14 \\
2(14.3)\end{array}$ \\
\hline Patients with first pregnancy & $26(63.4)$ & $17(70.8)$ & $9(45.0)$ \\
\hline Use of antihypertensive medication ${ }^{b}$ & $33(80.5)$ & $20(83.3)$ & $13(65.0)$ \\
\hline History of hypertension & $\begin{array}{l}N^{\mathrm{d}}=28 \\
16(57.1)\end{array}$ & $\begin{array}{l}N^{\mathrm{d}}=17 \\
10(58.8)\end{array}$ & $\begin{array}{l}N^{\mathrm{d}}=14 \\
8(57.1)\end{array}$ \\
\hline Blood pressure, $\mathrm{mm} \mathrm{Hg}$, median $(\text { range })^{\mathrm{c}}$ & $N^{\mathrm{d}}=20$ & $N^{\mathrm{d}}=10$ & $N^{\mathrm{d}}=10$ \\
\hline Systolic & $123(90-158)$ & $127(109-154)$ & $119(90-158)$ \\
\hline Diastolic & $71(60-95)$ & $73(63-93)$ & $71(60-95)$ \\
\hline History of dialysis & $25(60.1)$ & $15(62.5)$ & $12(60.0)$ \\
\hline Serum creatinine ${ }^{c}, \mu \mathrm{mol}$, median (range) & $\begin{array}{l}N^{\mathrm{d}}=17 \\
64(42-200)\end{array}$ & $\begin{array}{l}N^{\mathrm{d}}=9 \\
64(44-200)\end{array}$ & $\begin{array}{l}N^{\mathrm{d}}=8 \\
65(42-94)\end{array}$ \\
\hline
\end{tabular}

Data presented as $n(\%)$ unless otherwise stated. Baseline data were collected at Registry enrollment, except for blood pressure and serum creatinine, which were collected specifically during pregnancy. Data were obtained for 44 pregnancies in 41 patients; some patients had information recorded from multiple pregnancies. aHUS, atypical hemolytic uremic syndrome. ${ }^{\text {a }}$ Defined as patients with at least 1 family member with aHUS. ${ }^{b}$ At any time. ${ }^{c}$ Includes only measurements taken during pregnancy. Variable timing but at the closest time prior to end of pregnancy. ${ }^{\mathrm{d}}$ Data are presented for all patients with available information; this number may be lower than the total cohort population.

Table 2. Summary of pathogenic variants identified in pregnant patients with aHUS by exposure to eculizumab

$\begin{array}{lll}\text { All patients } & \text { Pregnancy with } & \text { Pregnancy with } \\ (N=41) & \text { exposure to } & \text { no exposure to } \\ & \text { eculizumab }(n=24) & \text { eculizumab }(n=20)\end{array}$

Number tested for $\geq 5$ pathogenic variants with no pathogenic variant identified, $n(\%)$

Any pathogenic variant, $n(\%) \quad 20(48.8)$

$10(24.4) \quad 7(29.2) \quad 4(20.0)$

Anti-CFH antibody tested and positive, $n(\%)$

$2(4.9)$

Any pathogenic variant found or anti-CFH antibody, $n(\%)$

$21(51.2)$

$11(45.8)$

$2(8.3)$

$12(50.0)$

$9(45.0)$

0

$9(45.0)$

Data were obtained for 44 pregnancies in 41 patients; some patients had information recorded from multiple pregnancies. aHUS, atypical hemolytic uremic syndrome; $\mathrm{CFH}$, complement factor $\mathrm{H}$.

plant and/or dialysis during the pregnancy, and with available data on the length of term, 8 resulted in premature births (34.8\%) - 4 in eculizumab-exposed and 4 in nonexposed pregnancies. The median (range) gestational age was 37 (26-40) weeks (34 [26-39] weeks and 37 [3040] weeks in eculizumab-exposed and nonexposed pregnancies, respectively); the median (range) birth weight was 2.9 (0.7-4.0) $\mathrm{kg}(2.5$ [0.7-3.9] kg and 3.3 [1.3-4.0] kg in eculizumab-exposed and nonexposed pregnancies, respectively). Nine low-birth weight newborns $(<2.5 \mathrm{~kg})$ were recorded ( 6 and 3 in eculizumab-exposed and nonexposed pregnancies, respectively [36.0\% of patients with available data]). No fetal abnormalities or malformations in any of these newborns were documented.

Seven patients had a history of kidney transplant and/ or concomitant dialysis during pregnancy (Table 5). Live births were reported in 2 of 3 patients with concomitant dialysis during pregnancy (gestational age 34 and 36 weeks). The third patient had a spontaneous abortion at approximately 6 weeks gestation. Three of 6 patients with 
Table 3. Specific pathogenic variants in pregnancies with available data

\begin{tabular}{|c|c|c|c|c|c|c|}
\hline & \multicolumn{2}{|l|}{ All patients $(N=41)$} & \multicolumn{2}{|c|}{$\begin{array}{l}\text { Pregnancy with exposure to eculizumab } \\
(n=24)\end{array}$} & \multicolumn{2}{|c|}{$\begin{array}{l}\text { Pregnancy with no exposure to } \\
\text { eculizumab }(n=20)\end{array}$} \\
\hline & $\begin{array}{l}\text { pathogenic variant } \\
\text { tested for, }{ }^{\text {a } n}\end{array}$ & $\begin{array}{l}\text { pathogenic variant } \\
\text { identified, } n(\%)\end{array}$ & $\begin{array}{l}\text { pathogenic variant } \\
\text { tested for, } n\end{array}$ & $\begin{array}{l}\text { pathogenic variant } \\
\text { identified, } n(\%)\end{array}$ & $\begin{array}{l}\text { pathogenic variant } \\
\text { tested for, } n\end{array}$ & $\begin{array}{l}\text { pathogenic variant } \\
\text { identified, } n(\%)\end{array}$ \\
\hline $\mathrm{CFH}$ & 28 & $12(42.9)$ & 17 & $6(35.3)$ & 13 & $6(46.2)$ \\
\hline CFB & 28 & $6(21.4)$ & 18 & $5(27.8)$ & 12 & $1(8.3)$ \\
\hline $\mathrm{C} 3$ & 25 & $5(20.0)$ & 17 & $4(23.5)$ & 9 & $1(11.1)$ \\
\hline Thrombomodulin & 10 & $2(20.0)$ & 6 & $1(16.7)$ & 4 & $1(25.0)$ \\
\hline Anti-CFH antibody & 26 & $2(7.7)$ & 19 & $2(10.5)$ & 10 & 0 \\
\hline MCP & 23 & $1(4.3)$ & 15 & $1(6.7)$ & 9 & 0 \\
\hline $\mathrm{CFI}^{\mathrm{b}}$ & 28 & 0 & 17 & 0 & 13 & 0 \\
\hline DGKE & 6 & 0 & 4 & 0 & 2 & 0 \\
\hline
\end{tabular}

Data were obtained for 44 pregnancies in 41 patients; some patients had information recorded from multiple pregnancies. CFB, complement factor B; CFH, complement factor H; CFI, complement factor I; DGKE, diacylglycerol kinase epsilon; MCP, membrane cofactor protein. ${ }^{\mathrm{a}}$ Number of pregnancies with pathogenic variant data. ${ }^{\mathrm{b}}$ After the data cutoff, 1 patient was later confirmed to have a pathogenic variant in CFI.

Table 4. Pregnancy outcomes

\begin{tabular}{lccc}
\hline & $\begin{array}{l}\text { All pregnancies } \\
(N=44)\end{array}$ & $\begin{array}{l}\text { Pregnancy with } \\
\text { exposure to } \\
\text { eculizumab }(n=24)\end{array}$ & $\begin{array}{l}\text { Pregnancy with no } \\
\text { exposure to eculi- } \\
\text { zumab }(n=20)\end{array}$ \\
\hline Live birth, $n / N(\%)^{\mathrm{a}}$ & $29 / 34(85.3)$ & $15 / 17(88.2)$ & $14 / 17(82.4)$ \\
Birth weight, ${ }^{\mathrm{b}} \mathrm{kg}$, median (range) & $2.9(0.7-4.0)$ & $2.5(0.7-3.9)$ & $3.3(1.3-4.0)$ \\
Gestational age, ${ }^{\mathrm{c}}$ weeks, median (range) & $37(26-40)$ & $34(26-39)$ & $37(30-40)$ \\
Full-term births, $n / N(\%)^{\mathrm{d}}$ & $15 / 23^{\mathrm{e}}(65.2)$ & $5 / 9(55.6)$ & $10 / 14(71.4)$ \\
Premature births, $n / N(\%)^{\mathrm{d}}$ & $8 / 23^{\mathrm{e}}(34.8)$ & $4 / 9(44.4)$ & $4 / 14(28.6)$ \\
Elective termination, $n / N(\%)$ & $10 / 44(22.7)$ & $7 / 24(29.2)$ & $3 / 20(15.0)$ \\
Miscarriage, $n / N(\%)$ & $4 / 44(9.1)$ & $2 / 24(8.3)$ & $2 / 20(10.0)$ \\
Late fetal death, $n / N(\%)$ & $1 / 44(2.3)$ & 0 & $1(5.0)$ \\
\hline
\end{tabular}

Data were obtained for 44 pregnancies in 41 patients; some patients had information recorded from multiple pregnancies. ${ }^{a}$ Proportion calculated from total number of births excluding elective terminations. ${ }^{b}$ Missing data for 4 births; 2 pregnancies exposed to and 2 pregnancies not exposed to eculizumab. ${ }^{c}$ Missing data for 2 births, both from pregnancies exposed to eculizumab. ${ }^{\mathrm{d}}$ Proportion calculated from total number of live births with gestational age data available. ${ }^{\mathrm{e}}$ Four patients with a history of renal transplant and/or on dialysis during pregnancy were excluded.

a prior renal transplant reported live births (gestational age 32-34 weeks); the other 3 reported spontaneous abortions between 6 and 7 weeks gestation. All 4 patients who had live births received eculizumab during pregnancy (note that one of these 4 patients had a history of renal transplant and was on dialysis so is presented in both groups above; Table 5). Two of these 4 patients were already receiving eculizumab at the time of pregnancy one patient who had previously been treated with eculizumab required eculizumab again during pregnancy due to TMA recurrence; the fourth patient, on concomitant dialysis (no transplant history), required eculizumab because she had suffered acute kidney injury resulting in dialysis early in the pregnancy.

Elective terminations were reported in $10(22.7 \%)$ pregnancies (7/24 [29.2\%] eculizumab-exposed and 3/20 [15.0\%] nonexposed pregnancies); 6 of these occurred before 9 weeks gestation. Where given, reasons cited were maternal health concerns $(n=5)$ and patient choice $(n=$ 4; Table 6).

Four $(9.1 \%)$ pregnancies resulted in miscarriage (Table 4), 2 each in eculizumab-exposed pregnancies ( 1 at 
Table 5. Pregnancies in patients with prior kidney transplants or dialysis during pregnancy

\begin{tabular}{|c|c|c|c|c|}
\hline History & Pregnancy outcome & $\begin{array}{l}\text { Gestational } \\
\text { age/timing }\end{array}$ & Pathogenic variant & $\begin{array}{l}\text { Eculizumab } \\
\text { during } \\
\text { pregnancy? }\end{array}$ \\
\hline History of renal transplant & Live birth & 32 weeks & Anti-CFH antibody & Yes \\
\hline History of renal transplant & Live birth & 34 weeks & Unknown & Yes \\
\hline History of renal transplant & Spontaneous abortion & $\sim 7$ weeks & No & Yes \\
\hline History of renal transplant & Spontaneous abortion & $\sim 6$ weeks & No & Yes \\
\hline On dialysis during pregnancy, history of renal transplant & Live birth & 34 weeks & $\mathrm{MCP}, \mathrm{CFH}$ & Yes \\
\hline On dialysis during pregnancy, history of renal transplant & Spontaneous abortion & $\sim 6$ weeks & $\mathrm{CFH}$ & No \\
\hline On dialysis during pregnancy, no history of renal transplant & Live birth & 36 weeks & Unknown & Yes \\
\hline
\end{tabular}

$\mathrm{CFH}$, complement factor $\mathrm{H}$; MCP, membrane cofactor protein.

Table 6. Elective terminations: gestational period and reasons

\begin{tabular}{|c|c|c|c|}
\hline \multicolumn{2}{|c|}{ Pregnancy with eculizumab exposure $(n=24)$} & \multicolumn{2}{|c|}{ Pregnancy without eculizumab exposure $(n=20)$} \\
\hline gestational period & reason & gestational period & reason \\
\hline$\sim 6$ weeks & Maternal health concerns & $8-9$ weeks & No reason given \\
\hline 7 weeks & Patient choice & $8-9$ weeks & Maternal health concerns \\
\hline 7 weeks & Patient choice & 4 months & Maternal health concerns \\
\hline 9 weeks & Patient choice & & \\
\hline$<12$ weeks & Patient choice & & \\
\hline 14 weeks & Maternal health concerns & & \\
\hline NA & Maternal health concerns & & \\
\hline
\end{tabular}

NA, not available.

7 weeks gestation, the other undisclosed) and nonexposed pregnancies (at 5 and 9 weeks gestation). One in utero fetal death occurred (Table 4); the patient was not exposed to eculizumab during the pregnancy and hypertension was the only disease-related abnormality recorded before and during pregnancy. At 19 weeks gestation, she developed acute TMA with placental infarction resulting in fetal death and commenced eculizumab shortly afterward. No neonatal malformations were reported.

\section{TMA Events}

Four patients each reported 1 TMA event during pregnancy, with only 1 receiving eculizumab prior to the TMA event (Fig. 2; patient 2). In this patient, TMA complications in the third trimester of her second pregnancy led to an emergency cesarean section and a premature, otherwise healthy baby was delivered at 26 weeks gestation. This patient had severe disease and previously had a pregnancy resulting in fetal death (while not on eculizumab [described before]). Delivery complications included ma- ternal hypertension. A pathogenic variant was confirmed (CFH c.3134 5T $>\mathrm{C}$ in intron 20).

Three patients not initially exposed to eculizumab, subsequently received eculizumab to treat TMA complications. Two patients experienced TMA events during the second trimester, and one underwent elective termination on medical advice ( 14 weeks' gestation) after being hospitalized for worsening aHUS and considered highrisk of maternal death due to pulmonary hypertension. A pathogenic variant in thrombomodulin was identified. The second patient was hospitalized at 11 weeks gestation due to aHUS recurrence and commenced eculizumab the following day. A healthy baby was delivered by cesarean section at 34 weeks. Pathogenic variants in $\mathrm{CFH}$ and membrane cofactor protein were identified. The third patient experienced postpartum TMA the day after placental abruption and cesarean section delivery of a premature, otherwise healthy baby. She experienced a postpartum hemorrhage and suspected aHUS recurrence with signs of hemolysis. Eculizumab had been discontinued 2.5 years earlier due to clinical improvement but was re- 


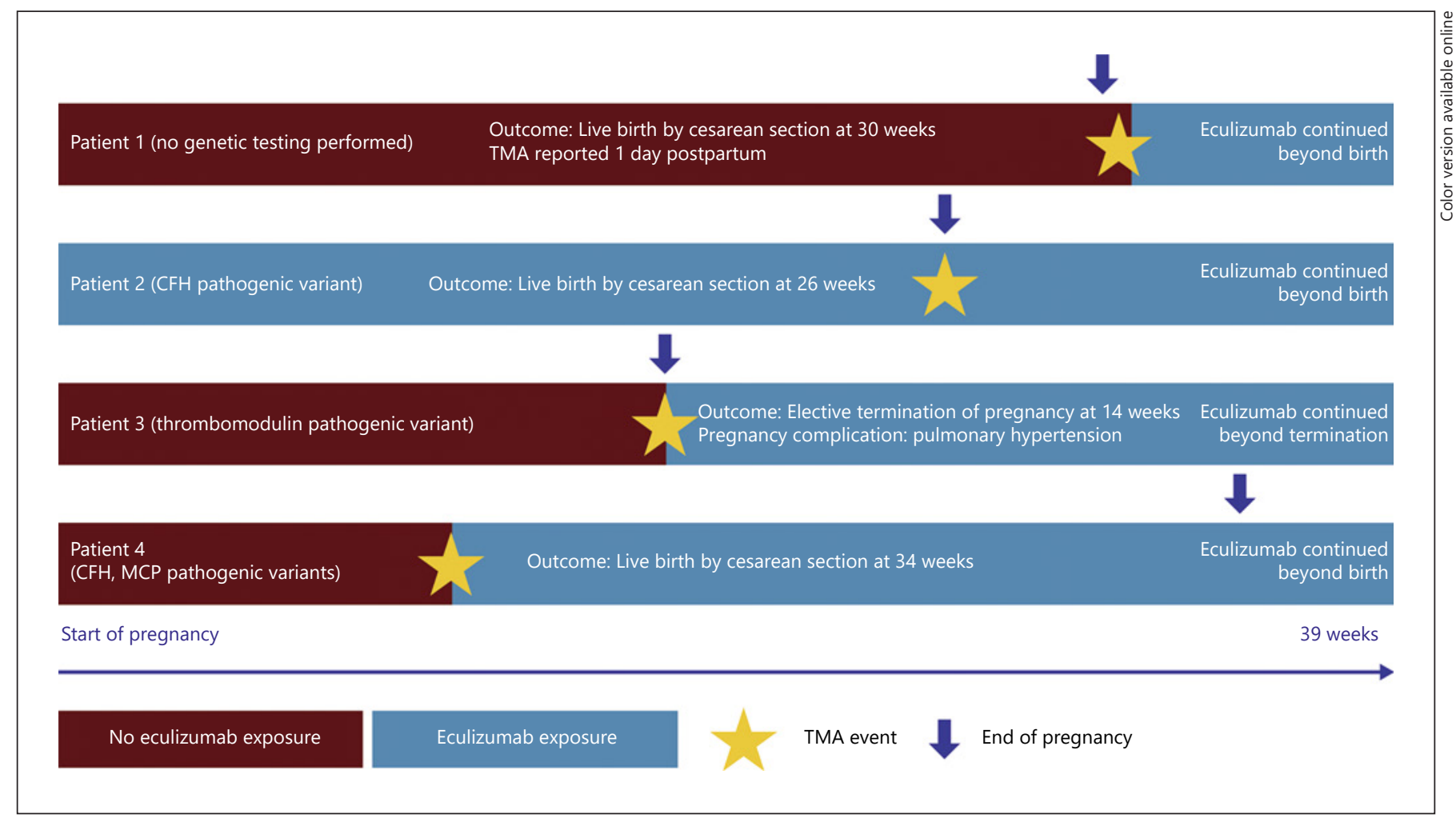

Fig. 2. Depiction of TMA complications during pregnancy that occurred in 4 patients. Patient 1 had had one prior pregnancy off eculizumab that was electively terminated. Patient 2 had had one prior pregnancy off eculizumab that resulted in fetal death. Patients 3 and 4 had not had any prior pregnancies. CFH, complement factor $\mathrm{H}$; MCP, membrane cofactor protein; TMA, thrombotic microangiopathy.

started on suspicion of aHUS relapse and was ongoing at last data entry. This patient had a pathogenic variant of CFI reported after the data cut.

\section{Discussion}

Here, we evaluated the characteristics and outcomes in women with a diagnosis of aHUS who subsequently became pregnant. Excluding elective terminations, the proportion of live births reported in this study $(85.3 \%)$ is comparable to recently reported pharmacovigilance data in patients with aHUS (74.4\%) [7] and mirrors findings in 6 pregnancies reported in patients with aHUS in the French registry [10]. The proportion of live births in pregnancies exposed (88.2\%) and not exposed (82.4\%) to eculizumab was comparable (with no reported fetal abnormalities).

In our study, the occurrence of miscarriage $(9.1 \%$ of all pregnancies) was numerically lower than reported in the pharmacovigilance study for patients with aHUS (14.8\%)

Pregnancy Outcomes in Women with Atypical Hemolytic Uremic Syndrome exposed to eculizumab [7] and was comparable to the general population, albeit without statistical comparison. A 2-year prospective community study in England found that $12 \%$ of 657 pregnancies miscarried before the 20th week [13], and according to the Center for Disease Control and Prevention in the USA, in 2008, approximately $17 \%$ of $6,578,000$ pregnancies ended in fetal loss (of all gestational periods) [14]. In our study, fetal loss (miscarriage plus late fetal death) occurred in $11.4 \%$ of pregnancies.

Approximately $23 \%$ of pregnancies in this study resulted in elective terminations, and from narrative descriptions of each case, the majority were likely driven by the perceived risks or fear of potential complications. These results are similar to those reported by the pharmacovigilance study in patients with aHUS (20.4\%) exposed to eculizumab [7]. Worldwide, in the general population between 2010 and 2014, 25\% of pregnancies ended in termination [15].

In this analysis, birth weight was similar to that in previous reports [8]. Low birth weight $(<2.5 \mathrm{~kg})$ was record- 
ed in $36 \%$ of pregnancies with available data. This is higher than that reported in a recent meta-analysis, where $21 \%$ of babies born to women with CKD were small for gestational age or had a low birth weight [16]. In addition, according to the Center for Disease Control and Prevention in the USA, $8 \%$ of infants were born with low birth weight and $1.4 \%$ with very low birth weight in 2017 [17]. The higher proportion of low-birth weight newborns in this study reflects the proportion born prematurely (44\%; 12 of 27 live births with data available), which is higher than that in the general population of the USA in 2017 (10\%) [17], global estimates in 2014 (11\%) [18], and CKD patients (24.2\%) [16]. This is consistent with data from other studies in patients with aHUS $[8$, $11,19]$.

The current data, alongside other reports, suggest that pregnancy is possible in patients with aHUS with careful monitoring. The foremost risk identified in patients with aHUS was preterm delivery. A previous study by Duval et al. [8], followed the progeny of 8 patients with aHUS over the first year and, despite being initially premature, all babies had a good prognosis.

The risk of disease recurrence during pregnancy is another concern for patients with aHUS affecting up to $20 \%$ of patients (mostly postpartum). One patient experienced a breakthrough TMA during pregnancy while on eculizumab, but it should be noted the patient had severe disease, a pathogenic variant in $\mathrm{CFH}$ and previously suffered a traumatic pregnancy resulting in fetal loss while not on eculizumab. She delivered a premature, but otherwise healthy, baby at 26 weeks via cesarean section following the TMA.

This study also provides data on pregnancy outcomes in patients on dialysis or with a history of renal transplantation, adding to the limited available data $[8,19]$. Three patients were on concomitant dialysis during their pregnancies, with live births recorded in 2 , and a spontaneous abortion in one. Six patients had previously received kidney transplants, with live births in 3 and spontaneous abortions in 3. All 4 patients with a history of renal transplant or concomitant dialysis during pregnancies that resulted in a live birth (gestational age 32-36 weeks) received eculizumab. One of these patients had a history of transplantation and was on dialysis. These data reinforce previous publications showing that pregnancy with live birth is possible in transplant $[8,19]$ and dialysis [20].

Complement pathogenic variants were recorded in 20 (48.8\%) of patients with available data, with $\mathrm{CFH}, \mathrm{CFB}$, and $\mathrm{C} 3$ most commonly indicating that the patient popu- lation was comparable to previous aHUS cohorts [2]. Due to the way data are collected for this Registry, there was no additional information as to why some patients with documented pathogenic variants were not treated with eculizumab, treatment decisions were made by treating physicians.

This study has several limitations due to the nature of data collection in observational registries. These include probable selection bias where only patients with more severe disease were treated with eculizumab as well as information bias relating to missing or incomplete data entry and lack of follow-up in some instances. Additionally, confounding variables and clinical characteristics that may have contributed to pregnancy outcomes were not controlled for in this descriptive analysis. Furthermore, there were not sufficient data reported on functional complement deficiencies. There was a limitation in the availability of some data, particularly serum creatinine at the beginning of pregnancy. As such, this was a descriptive analysis of Registry data, and no formal hypothesis testing was conducted. Finally, the sample size of this study, while large in context of studies in patients with aHUS, is still relatively small. A well-designed comparator study with a larger sample size would yield the most robust conclusions.

\section{Conclusion}

This real-world study suggests that pregnancy is possible in women with aHUS. Live birth proportions were high, albeit with high rates of premature birth, miscarriage proportions were low, and elective terminations observed were likely driven by perceived fear of potential complications. Our results also show that eculizumab has a good safety profile and is effective during pregnan$c y$, either as a prophylactic or curative treatment, even in dialyzed or transplanted patients. No new safety concerns for eculizumab use during pregnancy were observed. Continuous monitoring at high-risk pregnancy clinics is still needed, and further prospective data collection and analysis of a larger patient sample would be beneficial.

\section{Acknowledgments}

The sponsor and investigators thank the patients and their families for their participation in and support for this clinical study. The authors would also like to thank all the Registry investigators who have contributed data and the members of the Global aHUS 
Registry: Christoph Licht, Véronique Frémeaux-Bacchi, Gema Ariceta, Larry Greenbaum, Sally Johnson, Franz Schaefer, Leonard Woodward, Masayo Ogawa, and Christoph Gasteyger - Scientific Advisory Board members, and Miquel Blasco (Spain), Donata Cresseri (Italy), Galina Generolova (Russia), Nicholas Webb (United Kingdom), Patricia Hirt-Minkowski (Switzerland), Natalya Lvovna Kozlovskaya (Russia), Danny Landau (Israel), AnneLaure Lapeyraque (Canada), Chantal Loirat (France), Christoph Mache (Austria), Michal Malina (Czech Republic), Leena Martola (Finland), Annick Massart (Belgium), Eric Rondeau (France), and Lisa Sartz (Sweden) - National Coordinators of the Global aHUS Registry. The authors would like to thank Ciaran Wright, PhD, and Carolyn Bowler, $\mathrm{PhD}$, of Bioscript Medical, Macclesfield, UK, for providing editorial and medical writing support with funding from Alexion Pharmaceuticals, Inc. The authors would like to thank Radha Narayan, PhD, Alexion Pharmaceuticals, Inc. for critical review of the manuscript.

\section{Statement of Ethics}

This was a multicenter study comprising many different sites of enrollment. Federal, provincial, and local regulations and International Conference on Harmonization guidelines, if relevant, required that approval was obtained from an Ethics Committee (EC)/IRB prior to participation of patients in research studies. Where required and prior to the study onset, the EC/IRB must have approved the protocol, informed consent, advertisements to be used for patient recruitment, and any other written information regarding this study to be provided to the patient or the patient's parents/legal guardian. The sites maintained and made available for review by the sponsor or its designee documentation of all EC/IRB approvals and of the EC/IRB compliance with International Conference on Harmonization Guidance E6: Good Clinical Practice, if relevant. All EC/IRB approvals were signed by the EC/IRB chairman or designee and identified the EC/IRB name and address, the clinical protocol by title and/or protocol number, and the date approval and/or favorable opinion was granted. The investigator conducted all aspects of this study in accordance with all national, provincial, and local laws of the pertinent regulatory authorities. A written informed consent was obtained from each patient prior to participation in the study. The sponsor or its designee could provide an informed consent template to the sites, if required. If the site made any institutionspecific modifications, the sponsor or its designee could review the consent prior to IRB/EC submission. The investigator or the sponsor would then submit the approved, revised consent to the appropriate IRB/EC for review and approval prior to the start of the study. If the consent form was revised during the course of the study, all active participating patients to whom the revision may have had an impact must have signed the revised form. Before recruitment and enrollment, each patient was given a full explanation of the study and was allowed time to read the approved informed consent form. Once the investigator was assured that the individual understood the implications of participating in the study, the patient was asked to give consent to participate in the study by signing the informed consent form. The investigator provided a copy of the signed informed consent to the patient. The original form was maintained in the study files at the site.

Pregnancy Outcomes in Women with Atypical Hemolytic Uremic Syndrome

\section{Conflict of Interest Statement}

E.R. reports personal fees from Alexion, outside the submitted work; G.A. reports personal fees and other from Alexion Pharma, during the conduct of the study; F.F. reports other from Alexion, during the conduct of the study; personal fees from Alexion, personal fees from Roche, personal fees from Achillion, personal fees from Novartis, and personal fees from Apellis, outside the submitted work; I.A., B.M., and M.C. are employees of Alexion; M.S. reports personal fees from Alexion, outside the submitted work.

\section{Funding Sources}

This analysis was funded by Alexion Pharmaceuticals, Inc., Boston, MA. Alexion Pharmaceuticals, Inc., was responsible for the collection, management, and analysis of information contained in the Global aHUS Registry. Alexion Pharmaceuticals, Inc., contributed to data interpretation, preparation, review, and approval of the manuscript for submission. All authors had full access to all the data in the study and had final responsibility for the decision to submit for publication.

\section{Author Contributions}

All authors contributed to conceptualization of the study, study protocol design, and data interpretation. E.R., G.A., F.F., and M.S. contributed to data acquisition. I.A., M.C., and B.M. contributed to data analysis. All authors critically reviewed the manuscript and approved the final version.

\section{Data Availability Statement}

Alexion will consider requests for disclosure of clinical study participant-level data provided that participant privacy is assured through methods like data de-identification, pseudonymization, or anonymization (as required by applicable law), and if such disclosure was included in the relevant study informed consent form or similar documentation. Qualified academic investigators may request participant-level clinical data and supporting documents (statistical analysis plan and protocol) pertaining to Alexion-sponsored studies. Further details regarding data availability and instructions for requesting information are available in the Alexion Clinical Trials Disclosure and Transparency Policy at https://alexion.com/our-research/research-and-development. Link to data request form (https://alexion.com/contact-alexion/ medical-information). 


\section{References}

1 Campistol JM, Arias M, Ariceta G, Blasco M, Espinosa L, Espinosa M, et al. An update for atypical haemolytic uraemic syndrome: diagnosis and treatment. A consensus document. Nefrologia. 2015;35(5):421-47.

2 Fakhouri F, Zuber J, Frémeaux-Bacchi V, Loirat C. Haemolytic uraemic syndrome. Lancet. 2017;390(10095):681-96.

3 Fremeaux-Bacchi V, Fakhouri F, Garnier A, Bienaimé F, Dragon-Durey MA, Ngo S, et al. Genetics and outcome of atypical hemolytic uremic syndrome: a nationwide french series comparing children and adults. Clin J Am Soc Nephrol. 2013;8(4):554-62.

4 Webster P, Lightstone L, McKay DB, Josephson MA. Pregnancy in chronic kidney disease and kidney transplantation. Kidney Int. 2017; 91(5):1047-56.

5 Cabiddu G, Castellino S, Gernone G, Santoro D, Moroni G, Giannattasio M, et al. A best practice position statement on pregnancy in chronic kidney disease: the Italian Study Group on kidney and pregnancy. J Nephrol. 2016;29(3):277-303.

6 Tong A, Jesudason S, Craig JC, Winkelmayer WC. Perspectives on pregnancy in women with chronic kidney disease: systematic review of qualitative studies. Nephrol Dial Transpl. 2015;30(4):652-61.

7 Socie G, Caby-Tosi MP, Marantz JL, Cole A, Bedrosian CL, Gasteyger C, et al. Eculizumab in paroxysmal nocturnal haemoglobinuria and atypical haemolytic uraemic syndrome: 10 -year pharmacovigilance analysis. Br J Haematol. 2019;185(2):297-310.

8 Duval A, Olagne J, Cognard N, Gautier Vargas G, Joly M, Perrin P, et al. Pregnancy in a kidney transplant woman under treatment with eculizumab for atypical hemolytic uremic syndrome: is it safe? Kidney Int Rep. 2019;4(5):733-9.

9 Ardissino G, Wally Ossola M, Baffero GM, Rigotti A, Cugno M. Eculizumab for atypical hemolytic uremic syndrome in pregnancy. Obstet Gynecol. 2013;122(2 Pt 2):487-9.

10 Servais A, Devillard N, Frémeaux-Bacchi V, Hummel A, Salomon L, Contin-Bordes C, et al. Atypical haemolytic uraemic syndrome and pregnancy: outcome with ongoing eculizumab. Nephrol Dial Transpl. 2016;31(12):2122-30.

11 Gupta M, Govindappagari S, Burwick RM. Pregnancy-associated atypical hemolytic uremic syndrome: a systematic review. Obstet Gynecol. 2020;135(1):46-58.

12 Licht C, Ardissino G, Ariceta G, Cohen D, Cole JA, Gasteyger C, et al. The global aHUS registry: methodology and initial patient characteristics. BMC Nephrol. 2015;16:207.

13 Everett C. Incidence and outcome of bleeding before the 20th week of pregnancy: prospective study from general practice. BMJ. 1997; 315(7099):32-4.

14 Ventura SJ, Curtin SC, Abma JC, Henshaw SK. Estimated pregnancy rates and rates of pregnancy outcomes for the United States, 1990-2008. Natl Vital Stat Rep. 2012;60(7): $1-21$.

15 Sedgh G, Bearak J, Singh S, Bankole A, Popinchalk A, Ganatra B, et al. Abortion incidence between 1990 and 2014: global, regional, and subregional levels and trends. Lancet. 2016;388(10041):258-67.

16 Zhang JJ, Ma XX, Hao L, Liu LJ, Lv JC, Zhang $\mathrm{H}$. A systematic review and meta-analysis of outcomes of pregnancy in CKD and CKD outcomes in pregnancy. Clin J Am Soc Nephrol. 2015;10(11):1964-78.

17 Statistics CfDCaPNCfH. Birthweight and gestation; 2017. Available from: https://www. cdc.gov/nchs/fastats/birthweight.htm.

18 Chawanpaiboon S, Vogel JP, Moller AB, Lumbiganon P, Petzold M, Hogan D, et al. Global, regional, and national estimates of levels of preterm birth in 2014: a systematic review and modelling analysis. Lancet Glob Health. 2019;7(1):e37-46.

19 Gaggl M, Aigner C, Csuka D, Szilágyi Á, Prohászka Z, Kain R, et al. Maternal and fetal outcomes of pregnancies in women with atypical hemolytic uremic syndrome. J Am Soc Nephrol. 2018;29(3):1020-9.

20 Andries G, Karass M, Yandrapalli S, Linder K, Liu D, Nelson J, et al. Atypical hemolytic uremic syndrome in first trimester pregnancy successfully treated with eculizumab. Exp Hematol Oncol. 2017;6:4. 